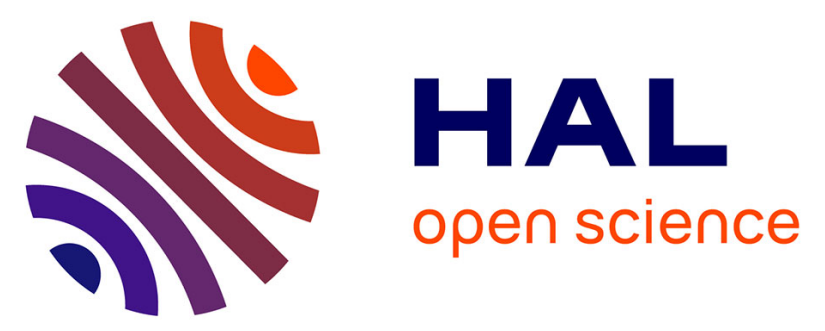

\title{
Impact of Physician Expertise on Probe Trajectory During Obstetric Ultrasound A Quantitative Approach for Skill Assessment
}

Maela Le Lous, Fabien Despinoy, Margaux Klein, Elisa Fustec, Vincent

Vincent.Lavoue@chu-Rennes.Fr Lavoué, Pierre Jannin

\section{To cite this version:}

Maela Le Lous, Fabien Despinoy, Margaux Klein, Elisa Fustec, Vincent Vincent.Lavoue@chuRennes.Fr Lavoué, et al.. Impact of Physician Expertise on Probe Trajectory During Obstetric Ultrasound A Quantitative Approach for Skill Assessment. Simulation in Healthcare, 2020, Publish Ahead of Print, 10.1097/SIH.0000000000000465 . hal-02887320v1

\section{HAL Id: hal-02887320 \\ https://hal.science/hal-02887320v1}

Submitted on 22 Oct 2020 (v1), last revised 4 Mar 2021 (v2)

HAL is a multi-disciplinary open access archive for the deposit and dissemination of scientific research documents, whether they are published or not. The documents may come from teaching and research institutions in France or abroad, or from public or private research centers.
L'archive ouverte pluridisciplinaire HAL, est destinée au dépôt et à la diffusion de documents scientifiques de niveau recherche, publiés ou non, émanant des établissements d'enseignement et de recherche français ou étrangers, des laboratoires publics ou privés. 
1 Impact of physician expertise on probe trajectory during obstetric ultrasound: a

2 quantitative approach for skill ass essment.

3 -Obstetrical Ultrasound Trajectory Metrics-

4

5 Maela LE LOUS, MD ${ }^{1,2}$, Fabien DESPINOY, $\mathrm{PhD}^{1}$, Margaux KLEIN, $\mathrm{MD}^{2}$, Elisa

6 FUSTEC $\mathrm{MD}^{2}$, Vincent LAVOUE MD $\mathrm{phD}^{2,3}$, Pierre JANNIN MD $\mathrm{PhD}^{1}$.

7

8

12 Corresponding author:

13 Maela Le Lous, MD

14 LTSI-INSERM, Université de Rennes 1, UMR 1099, 35000, Rennes, France.

15 Phone : Mobile +33695023805 Work + 3399263107

16 Fax

17 E-mail : maela.le.lous@chu-rennes.fr

18

19 Conflict of interest: The authors report no conflict of interest.

20 Funding: None

21 Word count 3298 ; Figure count : 2 ; Table count :2 


\section{Abstract}

23 Introduction The objective of the study was to identify objective metrics to evaluate the

24 significance of a sonographer's expertise on trajectories of ultrasound probe during 25 obstetric ultrasound training procedures.

26 Methods This Prospective observational study was conducted at Rennes University 27 Hospital, department of obstetrics and gynecology. We evaluated a panel of sonographers 28 (expert, intermediate and novice) in performing three tasks (Brain, Heart and Spine) with 29 an obstetric ultrasound simulator (Scantrainer, Medaphor ${ }^{\mathrm{TM}}$, Cardiff, UK). The 30 trajectories of the probe were logged and recorded by a custom data acquisition software.

31 We computed metrics on the trajectories (duration, path length, average velocity, average 32 acceleration, jerk, working volume) to compare the three groups and identify 33 discriminating metrics.

34 Results A total of 33 participants were enrolled: 5 experts, 12 intermediates, and 16 35 novices. Discriminatory metrics were observed among the three levels of expertise for 36 duration, velocity, acceleration and jerk for Brain and Spine tasks. Working volume was 37 discriminatory for the Brain and the Heart task. Path length was discriminatory for the 38 Brain task.

39 Conclusions Our results suggest a relationship between the sonographer's level of 40 expertise and probe trajectory metrics. Such measurements could be used as an indicator 41 of sonographer proficiency and contribute to automatic analysis of probe trajectory to 42 evaluate the quality of sonography and the sonographer.

44 Funding None.

45 Keywords Ultrasound; Obstetrics; Simulation training; Education; Standard setting. 


\section{Introduction}

Education in the field of obstetric ultrasound has shifted from an exclusively observation-based training to simulation-based training over the past decade ${ }^{1}$. Multiple factors may have contributed to the more widespread use of simulation: concerns for diagnostic errors, patient volunteers' or teachers' availability, and technological advances based on virtual reality (VR) ${ }^{23}$. Obstetric ultrasound simulators (OUS) may revolutionize the Obstetric and Gynecologic (OB/GYN) residents' curriculum for both training and evaluation ${ }^{4}$

The utility of OUS for ultrasound training is now well supported by evidence $5,6,7,8$. In a series of eight Danish studies, Toslgaard et al. successively explored the learning curves for novices, examined how to improve the efficiency of training with the use of dyad practice, and explored if improvements were sustained over time ${ }^{9}$. They also demonstrated skill transfer to subsequent clinical training ${ }^{10}$. Finally, they demonstrated an improvement for the patients after simulation training, such as a decrease in discomfort and improvements in their perception of safety and their confidence ${ }^{10}$.

Evaluation of competency in obstetric ultrasound is a time-consuming process and requires pregnant volunteers who are willing to be used for training. One study has shown the potential of OUS as a substitute for evaluating trainees ${ }^{11}$. In this study, dexterity and quality of images obtained during evaluation were assessed by two independent examiners. However, dexterity was subjectively scored between 0 and 10 based on observation.

A fundamental issue for evaluation with OUS is the need for metrics. VR based simulators allow automatic recording of probe trajectories during training and evaluation. OUS could be a suitable tool to extract data from these probe trajectories 
70 for objective evaluation, and to identify metrics that may discriminate the level of 71 expertise of participants during their training. The aim of our study was to assess the 72 potential of OUS to identify objective metrics to measure expertise in 73 ultrasonography. 


\section{Methods}

\section{Participants}

The experimental set-up was conducted at the Department of Gynecology and Obstetrics at the University Hospital of Rennes. All training and assessments were carried out in an undisturbed environment. The participants were recruited in June 2018 and the study was conducted from July to September 2018. Approval was obtained from our local institutional review board.

The participants were divided into three groups based on their levels of experience: medical students (novice), OB/GYN residents (intermediate), and OB/GYN consultants (experts). All participants were recruited locally at the department of Gynecology and Obstetrics, and provided oral and written informed consent. The experts were professional who had every day practice on genuine patients (about 25 morphologic scans a week). The intermediates were interns who are familiar with routine examination scans (fetal movements, amniotic fluid, estimation of fetal weight, about 5 scans a week) but not with morphologic examination. The novices had absolutely no ultrasound experience. The participants in the 3 groups never practiced on the simulator before the study, as the simulator was bought right before study beginning. The medical program at the University of Rennes is a 6-year traditional curriculum, and the gynecology rotations are completed during the 2 final years. The Novices were recruited during their gynecology rotations. During their prior medical training, the students had completed courses in pelvic anatomy, and ultrasound theory, but had no hands-on training. The Intermediates included OB/GYN residents who had not graduated in ultrasound but who were familiar with ultrasound equipment. The Experts were $\mathrm{OB} / \mathrm{GYN}$ consultants who used ultrasound on a daily basis. 


\section{Virtual reality simulator}

Training and assessments were performed using a high-fidelity VR based

103 simulator (Scantrainer, Medaphor ${ }^{\mathrm{TM}}$, Cardiff, UK) designed for obstetric ultrasound

104 training. It is composed of a monitor, an abdominal probe similar to a real one, docked

105 into a Sigma haptic device, two screens and a computer (Figure 1). The haptic device

106 provides realistic force feedback, when operator applies pressure on the abdominal probe.

107 One monitor displays B-Mode 2D ultrasound pictures provided by the system and

108 obtained from real patients. The second monitor displays an animated illustration of the

109 probe position in a virtual patient.

\section{Tasks performed}

\section{Step 1: Identification of tasks}

113 Most fetal malformations are detected during the second trimester scan. This

114 scan examines the fetus in 11 planes as requested by the National Committee for

115 Prenatal Ultrasound ${ }^{12}$.

116 Tasks reflecting the standardized second trimester examination of the fetus were

117 selected to obtain a large sample of fetal anatomy screening, in particular tasks which

118 involved switching from transverse to coronal or sagittal planes. We selected the tasks

119 which would potentially reflect differences in ultrasound competences and provided the

120 most realistic view of the fetus in three consecutive standardized planes. For Task 1

121 (Brain at 24 weeks of gestation), participants were asked to successively obtain the

122 standardized view of the fetal head circumference, then the standardized plane of the

123 cerebellum, and finally a coronal view of the brain through the cavum of septum 
124 pellucidum. For Task 2 (Heart at 22 weeks of gestation), participants were asked to 125 successively obtain the standardized view of the four chambers, the view of the left

126 ventricle and the aorta, and the view of the right ventricle and the pulmonary artery. For

127 Task 3 (Spine at 22 weeks of gestation), participants were asked to obtain successively 128 the standardized frontal view of the kidneys, a sagittal view of the spine, and the 129 parasagittal view of the left diaphragm. Figure 2 shows a pictorial representation of the 130 three tasks. The participant judged himself that the view was exact and he froze the 131 image to finish exercise. All participants were asked to provide their age, gender, and years of clinical 133 experience, and were assigned an identifier to anonymize the data. They then received a 134 short introduction to the simulated setting, including how to operate the simulator and its 135 functions. They also received a course about the recommended fetal planes. All trainees 136 practiced 10 minutes in a "probe manipulation exercise" to familiarize themselves with

137 the device, and to check the expertise difference between the groups. The score of this 138 exercise was calculated by adding the scores of six multiple choice questions (pass: 1; 139 fail: 0) designed to test recognition of six different 3-dimensional geometric solids (cone, 140 pyramid, spiral, spheres, other complex geometric solids) by scanning them with the 2-D

141 probe. Technical assistance was provided during the simulator test, but no instructions,

142 feedback or time limit were provided. Each participant had to perform the three tasks 143 three times. 
147 developed with Scantrainer, Medaphor ${ }^{\mathrm{TM}}$, Cardiff, UK. The probe position frequency

148 sampling was $20 \mathrm{~Hz}$. Computation of all metrics and trajectory analysis were run on a

149 dedicated computer (Xeon E5-1650V4 @3.60GHz with 32Go RAM) using

150 Matlab_R2017b. The analysis was done following a previously published technique ${ }^{13}$. A

151 total of 6 selected metrics was analyzed:

152 1. Duration (D) corresponds to the execution time between the first time the

153 hand moves the probe, until it has been released at the end of the task. It is

154 measured in seconds (s).

155 2. Path Length (PL) represents the total distance travelled by the probe during

156 the execution of the task. It is measured in millimeters (mm).

3. Average Velocity (AV) corresponds to the average linear speed of the probe during the task. It is measured in $\mathrm{mm} \cdot \mathrm{s}^{-1}$.

4. Average Acceleration (AA) corresponds to the average instantaneous acceleration of the probe during the task. It is measured in $\mathrm{mm} \cdot \mathrm{s}^{-2}$.

5. Average Jerk (AJ) corresponds to the average jerk (derivative of the acceleration) during the task, also known as "smoothness" measure. It is measured in $\mathrm{mm} \cdot \mathrm{s}^{-3}$.

6. Working volume (WV) represents the volume of the convex hull for each trajectory. The convex hull of a trajectory is the smallest convex volume among those which contain it. It is measured in $\mathrm{mm}^{3}$.

One-way Anova-3 tests were conducted to detect differences between the three 
169 control alpha inflation $(\mathbf{p}<\mathbf{0 . 0 8})$. The participants were absolutely not told about

170 what the metrics measured were.

171 


\section{Results}

\section{Demographics} Participant demographics according to expertise level are presented in Table 1.

\section{Manipulation probe exercise}

176

For the manipulation probe exercise, out of a total of six points (six multiple choice questions, pass: 1 ; fail: 0 ), the Experts' mean score was 5.6 \pm 0.5 , the Intermediates' mean score was $4.7 \pm 1.2$, and the Novices' mean score was $3.4 \pm 1.2$ points $(\mathrm{p}=0.002)$.

\section{Table 1.}

\section{Trajectory Metrics}

Analysis of the probe trajectory metrics revealed significant differences for Tasks

The mean Duration(s) of the exercise differed between Novices, Intermediates, and Experts for Task 1 (respectively $157.5 \mathrm{~s} \pm 176.2$ versus $111.2 \mathrm{~s} \pm 110.3$ versus $35.6 \mathrm{~s}$

$186 \pm 16.3, \mathrm{p}<0.001)$, Task $3(112.8 \mathrm{~s} \pm 64.7$ vs. $82.3 \mathrm{~s} \pm 50.6$ vs. $35.5 \mathrm{~s} \pm 11.4 \mathrm{p}<0.001)$. For Task

$1872(109.9 \mathrm{~s} \pm 69.7$ vs. $103.7 \mathrm{~s} \pm 61.1$ vs. $51.7 \mathrm{~s} \pm 47.4 \mathrm{p}=0.009)$ it was not significant.

190 (Novices: $1442.3 \pm 1134.4$ vs. Intermediates: $1795.4 \pm 1071.9$ vs. Experts: $1005.7 \pm 409.7$,

$191 \mathrm{p}<0.001$ ) and for Task 3 (respectively, $1515.3 \pm 569.4$ vs. $1590.8 \pm 589.0$ vs. 1071.2 $192 \pm 638.3, \mathrm{p}=0.013)$. 

expertise for Task 1 and 3. For Task 1, average velocity was $19.2 \mathrm{~mm} / \mathrm{s} \pm 5.7$ for Novices vs. $19.5 \mathrm{~mm} / \mathrm{s} \pm 13.8$ for Intermediate and $29.5 \mathrm{~mm} / \mathrm{s} \pm 8.7$ for Experts $(\mathrm{p}<0.001)$, and for

199 Task 3 it was respectively $15.6 \mathrm{~mm} / \mathrm{s} \pm 5.2$ vs. $22.2 \pm 7.5$ vs. $29.1 \pm 8.6(\mathrm{p}<0.001)$. For Task

2002 , it was respectively $15.7 \mathrm{~mm} / \mathrm{s} \pm 6.4$ vs. $14.5 \pm 7.0$ vs. $20.1 \pm 6.4(\mathrm{p}=0.024$, NS).

201 Average Acceleration $\left(m m \cdot s^{-2}\right)$

202

203

204

205

206

207

208

209

210

211

212

213

214

215

216

217

218

The average acceleration was statistically different between the three groups for Task $1\left(137.0 \mathrm{~mm} . \mathrm{s}^{-2} \pm 48.2\right.$ vs. $138.6 \pm 105.1$ vs. $\left.228.9 \pm 59.8, \mathrm{p}<0.001\right)$, and for Task 3 $(109.5 \pm 37.0$ vs. $157.3 \pm 54.6$ vs. $225.7 \pm 80.6, \mathrm{p}<0.001)$, but not for Task $2(115.8 \pm 62.8$ vs. $101.7 \pm 40.1$ vs. $149.6 \pm 37.5, \mathrm{NS})$

Average Jerk $\left(m m . s^{-3}\right)$

Jerk was statistically different between the three groups for Task 1 ( $2755.7 \pm 1$ 357.0 vs. $2728.5 \pm 2283.5$ vs. 4 528.0 $\pm 1358.4, \mathrm{p}<0.001)$ and for Task 3 (2 154.7 \pm 845.5 vs. $3099.7 \pm 1300.4$ vs. $4378.1 \pm 1605.7$, p $<0.001$ ) increasing with level of expertise. For Task 2, there was no significant difference ( $2426.8 \pm 1886.4$ vs. $2012.1 \pm 716.7$ vs. 2 $929.7 \pm 761.1, \mathrm{NS})$.

\section{Working Volume $\left(\mathrm{mm}^{3}\right)$}

The total Working Volume covered was statistically different between the three groups for Task 1 (1 $118661.6 \mathrm{~mm}^{3} \pm 622487.1$ vs. $1438677.7 \pm 716948.1$ vs. 761 $105.7 \pm 603917.3 \mathrm{p}=0.002$ ).

For Task 2 (1 $755821.8 \pm 1318436.4$ vs. $1275117.0 \pm 674373.0$ vs. 825782.0 \pm 962 957.2, NS) and Task 3, the difference was not significant (1 $618668.3 \pm 624129.9$ vs. $1759287.7 \pm 1250355.0$ vs. $867504.4 \pm 717940.5$, NS). 


\section{Discussion}

Main Findings

This paper presents for the first time the use of metrics computed on probe trajectory during simulated fetal ultrasound with OUS to objectively assess the expertise

223 of the user and his/her dexterity. The main result was that objective metrics (duration, 224 acceleration, velocity and jerk) differed statistically according to the level of expertise in 225 two of the three tasks.

The time for each task (Duration) decreased as the level of expertise increased ranging from $35 \mathrm{~s}$ to $52 \mathrm{~s}$ for Experts and from $109 \mathrm{~s}$ to $157 \mathrm{~s}$ for Novices. The Path Length, 228 although significantly shorter for Experts in the "Brain" and "Spine" exercise, was not 229 significantly different for the "Heart" exercise. This might be explained by the fact that 230 the planes in the heart exercise are much more tightly bunched in a very small volume.

231 There must have been a task effect. The "heart" exercise must have been less discriminant 232 using trajectory metrics, because it implied tiny probe movements. The same could have 233 been argued for the "Brain" exercise because there isn't a lot a movement between the

234 head circumference and the transcerebellar view, but a lot a movement is necessary to 235 obtain the coronal view. It is to notice that some amount of initial movement among 236 experts, must be tied to translating positioning and movements with the model to recall 237 of positioning and movements with genuine patients.

238 Velocity and Acceleration were significantly higher with the increase in level of 239 expertise suggesting that experienced sonographers move from one plane to another with 240 a faster movement. Jerk, which is a derivative of Acceleration, may be interpreted as the 241 variation of acceleration, or how sudden the variations are. We could have assumed that 242 jerk would have been smaller and the gesture smoother for the experts, as it has been 
243 shown for surgical procedures ${ }^{14}$. However, during ultrasound examination, jerk was

244 higher for experts because they most often combined a quick translation followed by a

245 quick $90^{\circ}$ rotation of the probe to switch, for example, from a transverse view of the

246 kidney to a sagittal view of the spine. For the "Heart" exercise, average Jerk did not differ

247 between the 3 groups, with may be explained again with the small quantity of movements

248 between the different views. The Working Volume was smaller for the Experts than for

249 the Intermediates and Novices, implying that the Experts remained within the area of

250 interest. These results are similar to those observed by Zago et al about FAST (Focused

251 Assessment with Sonography in Trauma) examination. In this study, they also used hand

252 motion analysis to discriminate expertise, and found similar results : longer hand path and

253 higher working volume for the novices ${ }^{15}$. In our study, intermediates performed more like

254 novices than experts on many measures because they may not feel comfortable with

255 morphologic examination.

256 These data are all the more important in France where, for the first time next year,

257 the practical exam for students will be by OUS rather than on volunteers. Other developed

258 countries are following the same trend, i.e., relying more on assessment by OUS.

259 Objective metrics are thus required to respond to this move towards automatic and

260 objective evaluation. Recording the trajectories of the probe, and comparing them to

261 trajectories obtained with experts is an interesting way of evaluating students' level. In a

262 clinical diagnostic perspective, trajectory metrics, taken separately, may not be relevant

263 to measure performance. However, these metrics are a first step. We tested some metrics,

264 and not all of them will have clinical meaning, but they do have a kinematic meaning.

265 Some are obvious (duration) and some are not but may be discriminant (jerk, volume). It 
is part of the method to go from the identification of differences to explanation of the differences observed.

Madsen et al. also analyzed simulator-generated metrics on a high fidelity

269 ultrasound transvaginal simulator ${ }^{6}$. They evaluated a group of 16 ultrasound novices 270 along with a group of $12 \mathrm{OB} / \mathrm{GYN}$ consultants. The score was calculated by adding the 271 scores of the seven modules ( 0 , fail; 1, pass) for each participant. Of the 153 metrics, 48 272 reliably discriminated between levels of competence and demonstrated evidence of 273 construct validity. However, in that study, simulator-generated metrics were dichotomic, 274 marked either pass or fail, which is different from the present study with a continuous 275 variable.

276 Few other studies have analyzed simulator-generated metrics, and none have analyzed trajectories. Furthermore, most publications on simulator-generated metrics 278 focus on laparoscopy training ${ }^{1617}$. Jones $\mathrm{D}$ et al. conducted a study extracting data form a laparoscopic simulator. They suggested a relationship between the training level of the 280 surgeon and the forces imparted on the tissue during a laparoscopic simulation ${ }^{18}$. In 281 another study about laparoscopic training, Rivard et al. selected 36 individual metrics on 282 four tasks, including speed, motion path length, respect for tissue, accuracy, task-specific 283 errors, and successful task completion. Time and motion path length were significantly 284 different for all four tasks, and the other metrics for some of the tasks. They then used the 285 validated metrics to create summary equations for each task, which successfully 286 distinguished between the different experience levels ${ }^{16}$. Lastly, another study about 287 laparoscopic procedures explored the correlation between path length or smoothness and 288 outcome measures such as accuracy error, knot slippage, leakage, tissue damage, and 289 operating time ${ }^{19}$. In that study, no correlation was found between the metrics and surgery 
290 outcomes, except for operative time. Finally, in another study by Sánchez-Margallo, the

291 suturing performance was successfully assessed by the motion analysis method. They

292 demonstrated construct validity for the execution time and path length ${ }^{20}$.

293 To integrate a simulator in a training and assessment program, it is necessary to

294 demonstrate face, content and construct validity of that simulator. The construct validity

295 means the ability to discriminate between different levels of expertise. By opposition,

296 face and content validity means how convincing or realistic the simulator is according to

297 experts, in a more subjective way ${ }^{21}$. It is interesting to notice how objective measures are

298 used to inform construct validity. A study by Van Dongen et al. which aimed to

299 demonstrate construct validity for a laparoscopic virtual reality simulator, used the

300 clinical experience as a definition of expertise. Indeed, simulator metrics were tested in

30116 novices, 16 residents and 16 experts to construct content validity of a laparoscopic

302 simulator $^{22}$. It appeared that performance of the various tasks on the simulator

303 corresponded to the respective level of laparoscopic clinical experience.

304 In a study by Ramos et al, participants completed three virtual reality (VR)

305 exercises using the Da Vinci Skills Simulator, as well as corresponding dry lab versions

306 of each exercise. Simulator performance was assessed by metrics measured on the

307 simulator. Dry lab performance was blindly video-evaluated by expert review using the

308 six-metric GEARS tool. This study is interesting because their definition of expertise was

309 based on an exercise and not just the years of clinical experience. Additionally,

310 participants were ask to complete a questionnaire to evaluate face and content validity. ${ }^{23}$

311 In another study by Kenney et al, construct validity of a robotic surgery virtual-reality

312 trainer was assessed ${ }^{24}$. The performance was recorded using a built-in scoring algorithm

313 including total task time, total instrument motion, and number of instrument collisions. 
314 Experienced robotic surgeons outperformed novices in nearly all variables. Again, each

315 subject completed a questionnaire after finishing the modules to assess face and content

316 validity. All experienced surgeons ranked the simulator as useful for training and agreed

317 with incorporating the simulator into a residency curriculum.

Strengths and Limitations

One limitation of our study is linked with the definition of expertise. The three expertise levels were defined by the clinical experience, not the quality of the scans or images that the participants actually produced. However, clinical experience levels were clearly defined (experts who had everyday practice of fetal ultrasound, residents who

324 were much less experienced, and novices who were medical students with no experience at all). Moreover, to address the potential bias on how to define an expert, every participant achieved a probe manipulation exercise, which confirmed levels in the three groups. Another limitation is that we did not assess face and content validity, because we focused on objective metrics.

331 the fetus and how to switch from one plane to another, especially to sagittal views. Future

332 explorations are required to approach the optimal trajectory of the probe. This could help

333 to better teach the technique, optimize scan duration, and assess the dynamic quality of

334 the exploration. Future works should also assess skill transfer to clinical practice and 335 trajectories on actual patients. 
This study shows that objective trajectory metrics differ according to level of

340 expertise in two OUS tasks. The connected OUS interface between the operator's hand

341 and the patient, provides numerical data that can help better understand and assess skill

342 acquisition. It is the responsibility of the clinicians to let the developers know what data

343 they are interested in that would make the simulators suited for training. 


\section{Acknowledgements}

346 The authors would like to thank all participants in this study, Felicity Neilson for

347 English editing, and Stephen Thucker for technical support on the simulation system.

348

349 Disclos ure of interests:

350 The authors declare no conflict of interest.

351

352 Authorship contribution:

353 MLL carried out the study, analyzed the literature, and wrote up the paper.

354 FD conducted the analyze of the metrics.

$355 \mathrm{MK}$ and EF had a role in participants enrollment, helped with experiments and data

356 collection, read and corrected the paper.

357 VL read and added correction to the draft and had a role in the conception and

358 correction of the paper.

359 PJ had a role in the conception, planning, and correction of the paper.

360

361 Details of Ethics Approval :

362 Approval was obtained from our local institutional review board (University Hospital of

363 Rennes, IRB Approval Avis n 19.29, 03/03/2019).

364

365 Funding : None 


\section{References}

1. Cook DA. How much evidence does it take? A cumulative meta-analys is of outcomes of simulation-based education. Med Educ 2014; 48: 750-760.

2. Scalese RJ, Obeso VT, Issenberg SB. Simulation technology for skills training and competency assessment in medical education. J Gen Intern Med 2008; 23 Suppl 1: 46-49.

3. Scott DJ, Dunnington GL. The new ACS/APDS Skills Curriculum: moving the learning curve out of the operating room. J Gastrointest Surg 2008; 12: 213-221.

4. Tolsgaard MG. A multiple-perspective approach for the assessment and learning of ultrasound skills. Perspect Med Educ 2018; 7: 211-213.

5. Chalouhi GE, Quibel T, Lamourdedieu C, et al. [Obstetrical ultrasound simulator as a tool for improving teaching strategies for beginners: Pilot study and review of the literature]. J Gynecol Obstet Biol Reprod (Paris) 2016; 45: 1107-1114. 6. Madsen ME, Konge L, Nørgaard LN, et al. Assessment of performance measures and learning curves for use of a virtual-reality ultrasound simulator in transvaginal ultrasound examination. Ultrasound Obstet Gynecol 2014; 44: 693-699. 7. Tolsgaard MG, Rasmussen MB, Tappert C, et al. Which factors are associated with trainees' confidence in performing obstetric and gynecological ultrasound examinations? Ultrasound Obstet Gynecol 2014; 43: 444-451.

8. Tolsgaard MG, Chalouhi GE. Use of simulators for the assessment of trainees' competence: trendy toys or valuable instruments? Ultrasound Obstet Gynecol. Epub ahead of print 17 April 2018. DOI: 10.1002/uog.19071. 9. Tolsgaard MG, Ringsted C, Dreisler E, et al. Sustained effect of simulationbased ultrasound training on clinical performance: a randomized trial. Ultrasound Obstet Gynecol 2015; 46: 312-318.

10. Tolsgaard MG, Ringsted C, Rosthøj S, et al. The Effects of Simulation-based Transvaginal Ultrasound Training on Quality and Efficiency of Care: A Multicenter Single-blind Randomized Trial. Ann Surg 2017; 265: 630-637.

11. Chalouhi GE, Bernardi V, Gueneuc A, et al. Evaluation of trainees' ability to perform obstetrical ultrasound using simulation: challenges and opportunities. Am J Obstet Gynecol 2016; 214: 525.e1-525.e8.

12. cneof 2016 - Recherche Google, https://www.google.com/search?client=fire fox$\mathrm{b} \& \mathrm{q}=$ cneof +2016 (accessed 7 January 2019).

13. Despinoy F, Zemiti N, Forestier G, et al. Evaluation of contactless humanmachine interface for robotic surgical training. Int J Comput Assist Radiol Surg 2018; 13: $13-24$.

14. Ghasemloonia A, Maddahi Y, Zareinia K, et al. Surgical Skill Assessment Using Motion Quality and Smoothness. J Surg Educ 2017; 74: 295-305. 15. Zago M, Sforza C, Mariani D, et al. Educational impact of hand motion analysis in the evaluation of FAST examination skills. Eur J Trauma Emerg Surg. Epub ahead of print 15 March 2019. DOI: 10.1007/s00068-019-01112-6.

16. Rivard JD, Vergis AS, Unger BJ, et al. Construct validity of individual and summary performance metrics associated with a computer-based laparoscopic simulator. Surg Endosc 2014; 28: 1921-1928.

17. Shanmugan S, Leblanc F, Senagore AJ, et al. Virtual reality simulator training for laparoscopic colectomy: what metrics have construct validity? Dis Colon Rectum 2014; 57: 210-214. 
414 18. Jones D, Jaffer A, Nodeh AA, et al. Analysis of Mechanical Forces Used During 415 Laparoscopic Training Procedures. J Endourol 2018; 32: 529-533.

416 19. Cesanek P, Uchal M, Uranues S, et al. Do hybrid simulator-generated metrics

417 correlate with content-valid outcome measures? Surg Endosc 2008; 22: 2178-2183.

418 20. Sánchez-Margallo JA, Sánchez-Margallo FM, Oropesa I, et al. Objective

419 assessment based on motion-related metrics and technical performance in laparoscopic

420 suturing. Int J Comput Assist Radiol Surg 2017; 12: 307-314.

421 21. Koch AD, Buzink SN, Heemskerk J, et al. Expert and construct validity of the

422 Simbionix GI Mentor II endoscopy simulator for colonoscopy. Surg Endosc 2008; 22:

$423 \quad 158-162$.

424 22. van Dongen KW, Tournoij E, van der Zee DC, et al. Construct validity of the

425 LapSim: can the LapSim virtual reality simulator distinguish between novices and

426 experts? Surg Endosc 2007; 21: 1413-1417.

427 23. Ramos P, Montez J, Tripp A, et al. Face, content, construct and concurrent

428 validity of dry laboratory exercises for robotic training using a global assessment tool.

429 BJU Int 2014; 113: 836-842.

430 24. Kenney PA, Wszolek MF, Gould JJ, et al. Face, content, and construct validity

431 of dV-trainer, a novel virtual reality simulator for robotic surgery. Urology 2009; 73:

$432 \quad 1288-1292$.

433 


\section{Legends}

435 Figure 1. The virtual reality ultrasound simulator (Scantrainer, Medaphor ${ }^{\mathrm{TM}}$, Cardiff, 436 UK).

437 Figure 2. Pictorial representation of the three tasks. 